ITC 2/50

Information Technology and Control

Vol. 50/ No. 2/ 2021

pp. 342-356

DOI 10.5755/j01.itc.50.2.28087
Convolutional-Neural-Network Assisted Segmentation and SVM Classification of Brain Tumor in Clinical MRI Slices

Received 2020/11/26

Accepted after revision 2021/02/02

SS

HOW TO CITE: Rajinikanth, V., Kadry, S., Nam, Y. (2021). Convolutional-Neural-Network Assisted Segmentation and SVM Classification of Brain Tumor in Clinical MRI Slices. Information Technology and Control, 50(2), 342-356. https://doi.org/10.5755/j01.itc.50.2.28087

\title{
Convolutional-Neural-Network Assisted Segmentation and SVM Classification of Brain Tumor in Clinical MRI Slices
}

\section{Venkatesan Rajinikanth}

Department of Electronics and Instrumentation Engineering, St. Joseph's College of Engineering, Chennai 600119, India; e-mail: v.rajinikanth@ieee.org

\section{Seifedine Kadry}

Department of Mathematics and Computer Science, Faculty of Science, Beirut Arab University, Lebanon; e-mail: skadry@gmail.com

\section{Yunyoung Nam}

Department of Computer Science and Engineering, Soonchunhyang University, Asan, South Korea; e-mail:ynam@sch.ac.kr

\section{Corresponding author: ynam@sch.ac.kr}

Due to the increased disease occurrence rates in humans, the need for the Automated Disease Diagnosis (ADD) systems is also raised. Most of the ADD systems are proposed to support the doctor during the screening and decision making process. This research aims at developing a Computer Aided Disease Diagnosis (CADD) scheme to categorize the brain tumour of 2D MRI slices into Glioblastoma/Glioma class with better accuracy. The main contribution of this research work is to develop a CADD system with Convolutional-Neural-Network (CNN) supported segmentation and classification. The proposed CADD framework consist of the following phases; (i) Image collection and resizing, (ii) Automated tumour segmentation using VGG-UNet, (iv) Deep-feature extraction using VGG16 network, (v) Handcrafted feature extraction, (vi) Finest feature choice by firefly-al- 
gorithm, and (vii) Serial feature concatenation and binary classification. The merit of the executed CADD is confirmed using an investigation realized using the benchmark as well as clinically collected brain MRI slices. In this work, a binary classification with a 10-fold cross validation is implemented using well known classifiers and the results attained with the SVM-Cubic (accuracy >98\%) is superior. This result confirms that the combination of CNN assisted segmentation and classification helps to achieve enhanced disease detection accuracy. KEYWORDS: Brain tumour, MRI, VGG16, VGG-UNet, Firefly-algorithm, SVM-Cubic classifier.

\section{Introduction}

Due to various uncontrollable reasons, the disease occurrence rates in humans are gradually growing worldwide. The diseases in internal body organs are considered more acute compared to the diseases in external organs. In human physiology, the brain is one of the vital internal organ and also the prime part in Central Nervous System (CNS) and the abnormality/ disease in brain is one of the chief medical emergency [1-3]. The brain abnormality is due to various reasons and the brain tumour is one of the leading causes of abnormality in CNS.

The various class of Brain Tumour (BT) based on the dimension and the orientation is clearly discussed in [4] and this report also suggests various clinical treatment procedures for the BT, such as surgery, radiation therapy, and chemotherapy. In humans, the tumours, such as Low-Grade-Glioma (LGG) and Glioblastoma-Multiforme (GBM) causes a severe problem in CNS and the efficient recognition of these tumour cases are very essential to plan and implement appropriate treat process. The LGG begins in glial-cells of the CNS and rigorously affects the normal activity of CNS based on the orientation and progression rate. The GBM is one of the harsh condition, normally occurs in the chief part of the CNS (brain/ spinal cord) and the untreated GBM may lead to various problems, like headaches, nausea, vomiting and seizures [5-7].

The screening of the BT can be performed using signal-assisted-methodologies (recording and examining the electroencephalogram) and image-assisted-procedures (recording the brain section using radiological imaging). The $\mathrm{BT}$ detection with imaging practice helps to reach a healthier diagnosis compared to signal supported techniques [8-10].

In the literature, a number of image processing procedures are proposed and implemented to evaluate the BT using MRI slices of varied modalities. The MRI slice with axial-view is widely considered by the re- searchers and doctors to evaluate the abnormality, since the brain section seen in the axial-view is clear compared to sagittal- and coronal-view [11,12].

The clinical level detection of the BT using the chosen MRI slice is a challenging task during the mass screening operation and classifying the BT into GBM/LGG is necessary during the decision making and treatment planning process. When, the number of MRI slices to be diagnosed increases, then a Computer-Aided-Detection (CAD) can be implemented to reduce the burden and the report obtained from the CAD along with the categorized images are submitted for doctor's perusal. The CAD report combined with the findings by the doctor will help in taking the firm decision regarding the $\mathrm{BT}$ class and the possible treatment procedure to be implemented to recover the patient.

The proposed research aims at developing an appropriate Computer Aided Disease Diagnosis (CADD) scheme to segment and categorize the BT existing in the T2 modality brain MRI slice. The proposed CADD framework consist of the following stages; (i) Two-dimensional slice extraction and resizing (224x224x3 pixels), (ii) Deep-Feature (DF) extraction with VGG16, (iii) VGG-UNet assisted tumour mining, (iv) Handcrafted-feature (HF) extraction, (v) Dominant feature selection using Firefly-Algorithm, (vi) Serial features concatenation, and (vii) Binary classification with a 10 -fold cross confirmation.

The performance of the proposed CADD is tested and authenticated using benchmark as well as clinically collected MRI. The main purpose of the benchmark images (T2 modality MRI slices with LGG/GBM) is to train the VGG16 and VGG-UNet for the proposed task. The result attained with the proposed study confirms that the developed CADD helps in achieving a better accuracy with the benchmark (96.04\%) as well as clinical images (98.89\%). 
The main contribution of this research includes:

1 Implementation of CNN supported segmentation and classification for the brain MRI assessment;

2 Extracting the deep as well as all the possible handcrafted features;

3 Firefly algorithm supported dominant feature selection and binary classification.

This research is prepared as follows; Section 2 presents the context, Section 3 discusses the implemented methodology, Section 4 and 5 depicts the experimental results and discussions. The conclusion of this research is specified in Section 6.

\section{Related Works}

Due to its significance, a significant number of BT diagnosis systems are proposed and applied by the researchers using, traditional, Machine-Learning (ML) and Deep-Learning (DL) approaches [1-3]. In most of the CAD schemes, ML/DL methods are applied to classify the brain MRI slices based on the disease conditions.

The ML based BT detection directly implements a scheme with the following phases; MRI processing and resizing, feature extraction, feature selection, classifier implementation and validation [12]. In few existing ML technique, the extraction and evaluation of the BT section is also presented [14]. The ML approaches implemented in earlier research offered a satisfactory classification result on the benchmark as well as the clinical grade MRI slices (94.51\%) when a binary classification is employed to categorize the MRI slices into healthy/disease class [10]. The DL supported approaches helped to achieve a better result during the binary as well as the multi-class categorization of the brain MRI slices [7,8]. The earlier works on BT detection confirmed that, the DL schemes implemented with the DF and combined DF and $\mathrm{HF}(\mathrm{DF}+\mathrm{HF})$ will offer a better detection accuracy. The works presented in [11] evident the need for $\mathrm{DF}+\mathrm{HF}$ in order to enhance the disease detection.

The recent work discussed in [11] implemented a serial concatenation of DL and HF to enhance the performance of VGG19 architecture and the implemented technique helps in achieving a better classification accuracy with benchmark (98.00\%) and clinical database (98.17\%) MRI slices of T2 modality. The existing works in the literature [15-17] confirmed the need for combining the segmentation, $\mathrm{HF}$ extraction and DF extraction methodologies to enhance the overall accuracy of the disease detection system.

Hence, in this work a CADD framework is developed by combining the automated segmentation and classification scheme to improve the BT categorization accuracy and the proposed work is experienced and authenticated using the benchmark and clinical grade brain MRI slices of T2 modality. In this work, a pre-trained VGG16 architecture is considered and its segmentation/classification performance is initially trained, tested and validated using the TCIA dataset with GBM/ LGG image cases. Later, the BT detection performance of VGG16 is then confirmed using the clinically collected brain MRI slices. The attained result with the proposed CADD confirms that, the implemented VGG16 architecture offers a better BT detection accuracy.

In the earlier works, brain MRI segmentation and classification [9] is seperately discussed by the researchers. Further, the existing brain MRI slices are classified using the machine-learning [10] and/or deep-learning [11] methods. The chied motivation of the proposed research is to implement the CNN based joint segmentation and classifcation to enhance the disease detection accuracy for the benchmark as well as the clinical grade images.Further, to improve the classification accuracy, the optimally selected handcrafted features are combined with the deep-features and a binary classifier is implemented to categorize the images. This work aimed to implement a novel CADD system to classifiy the brain abnormality into LGG/GBM class using the benchmark as well as the clinically obtained MRI slices of real patients.

\section{Methodology}

The disease detection performance of every CAD unit depends on the tactic considered to execute the particular system. In this work, the BT detection is achieved using a novel CADD unit developed and implemented using the pre-trained Convolutional-Neural-Network (CNN) scheme.

\subsection{Disease Detection Framework}

The CADD unit considered to detect and categorize the BT of brain MRI slices is depicted in Figure 1. 
Figure 1

Proposed CADD framework to examine brain MRI slices

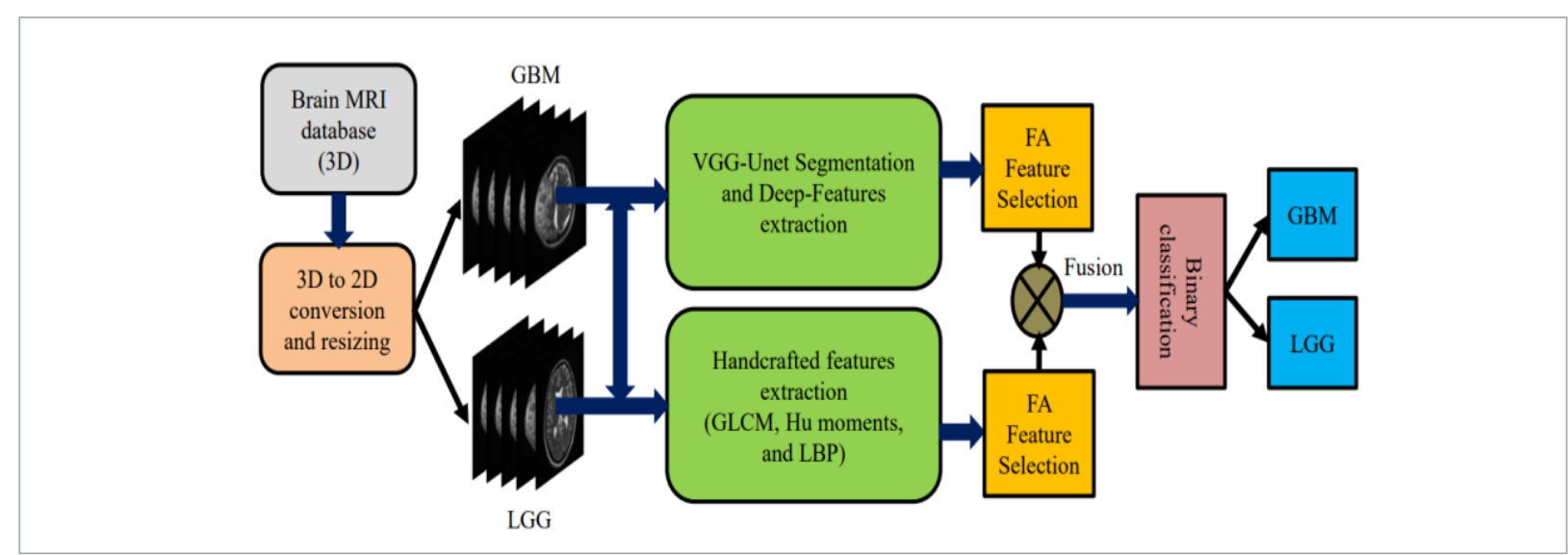

Initially, a 3D image of the brain MRI is collected from the patients and then a 3D to 2D conversion is implemented using ITK-Snap software [18]. The extracted 2D slices are then resized to $224 \times 224 \times 3$ pixels (recommended image dimension for VGG16). The resized images are then considered for the feature extraction task. The BT segment and the DF are extracted by employing a trained VGG-UNet and the $\mathrm{HF}$ are extracted using methods, such as GLCM, Hu, and LBP with different weights. After extracting the essential features, the dominant feature vector for $\mathrm{DF}$ and HF is selected using the Firefly-Algorithm and the selected features are sorted and combined using the serial feature concatenation technique discussed in [11]. The concatenated features are then considered to train, test and validate the binary classifiers, which helps to categorize the brain MRI slices into GBM and LGG class.

\subsection{Image Database}

The proposed work considers T2 modality brain MRI slices of GBM/LGG for the assessment and during this work; both the benchmark as well as clinically collected dataset are considered.

\subsubsection{Benchmark Database}

The Cancer Imaging Archive (TCIA) [19] is one of the vital data-source widely adopted by the researchers to evaluate their disease detection systems. In this work, the essential GBM [20] and LGG [21] classes of brain images with T2 modality are collected for the assessment. These images are available in 3D form and the 2D slices are then extracted with ITK-Snap and resized to $224 \times 224 \times 3$. The sample test images adopted in this work is depicted in Figure 2. The total number of images considered for GBM/LGG is presented in Table 1.

Figure 2

Sample test images collected from TCIA database
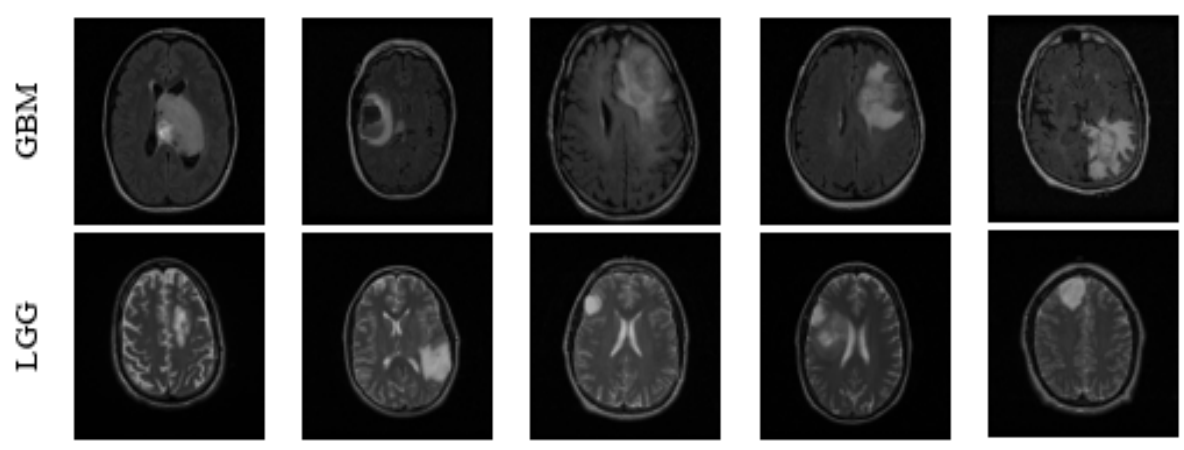
Table 1

TCIA and clinical-grade brain MRI slices considered in this study

\begin{tabular}{c|c|c|c|c}
\hline \multirow{2}{*}{ Image class } & Image & & \multicolumn{2}{|c}{ Brain MRI Slices } \\
\hline modality & Dimension & Training & Validation \\
\hline TCIA-GBM & T2 & $224 \times 224 \times 3$ & 560 & 240 \\
\hline TCIA-LGG & T2 & $224 \times 224 \times 3$ & 560 & 240 \\
\hline Clinical-GBM & T2 & $224 \times 224 \times 3$ & 210 & 90 \\
\hline
\end{tabular}

Figure 3

Sample T2 modality brain MRI slices of clinical database
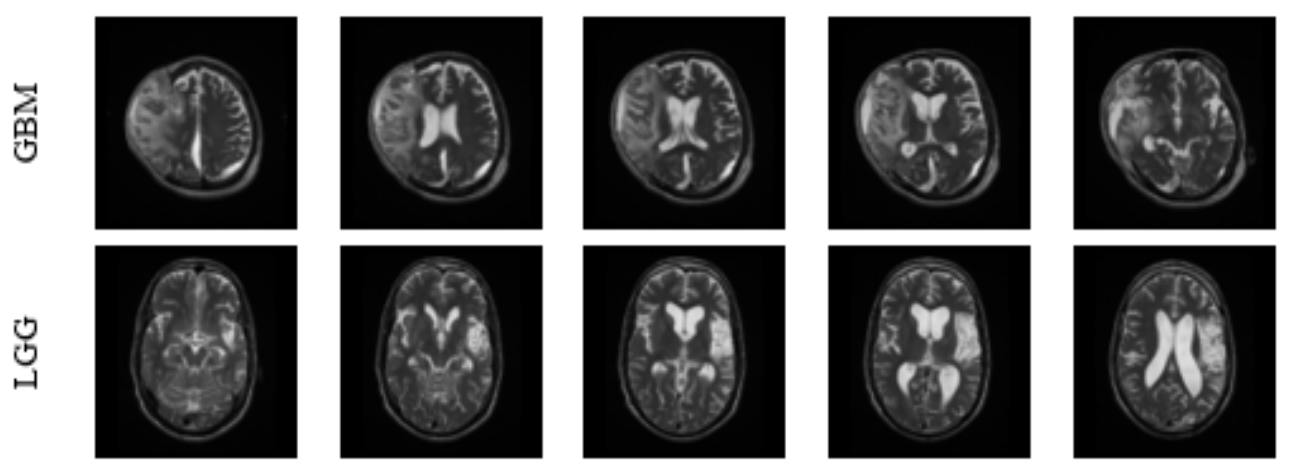

\subsubsection{Clinical Database}

The clinical significance of the proposed CADD unit is confirmed by considering the clinically collected real patient's MRI slices of T2 modality. The earlier works implemented with this dataset can be accessed from [9-11]. All the real patient's images are collected based on the approved medical protocol and informed consent is obtained from each patient participated in this study and all this information can be found in [10]. The sample clinical images of GBM/LGG can be found in Figure 3 and number of MRI slices considered in this work is available in Table 1.

\subsection{CNN Segmentation and Feature Extraction}

The image segmentation is one of the proven approaches, widely adopted to extract the abnormal section from the test image for further assessment
$[22,23]$. Automated segmentation is widely adopted compared to semi-automated and traditional procedures and hence, in the proposed work, CNN supported segmentation is implemented to extract the BT segment from the considered brain MRI slices.

\subsection{VGG-UNet Implementation}

The automated segmentation using UNet is initially proposed in [24]. This scheme included a encoder-decoder section to categorize the image components based on its pixel and for the medical image assessment, a binary classification is employed to extract the abnormal section. In this work, the VGG-UNet scheme depicted in Figure 4 is employed to extract the BT with better accuracy. The essential information on VGG-UNet can be accessed from [25-27]. The initial part (encoder) of VGG-UNet consists of the 
Figure 4

VGG-UNet implemented to extract the tumour from MRI slices

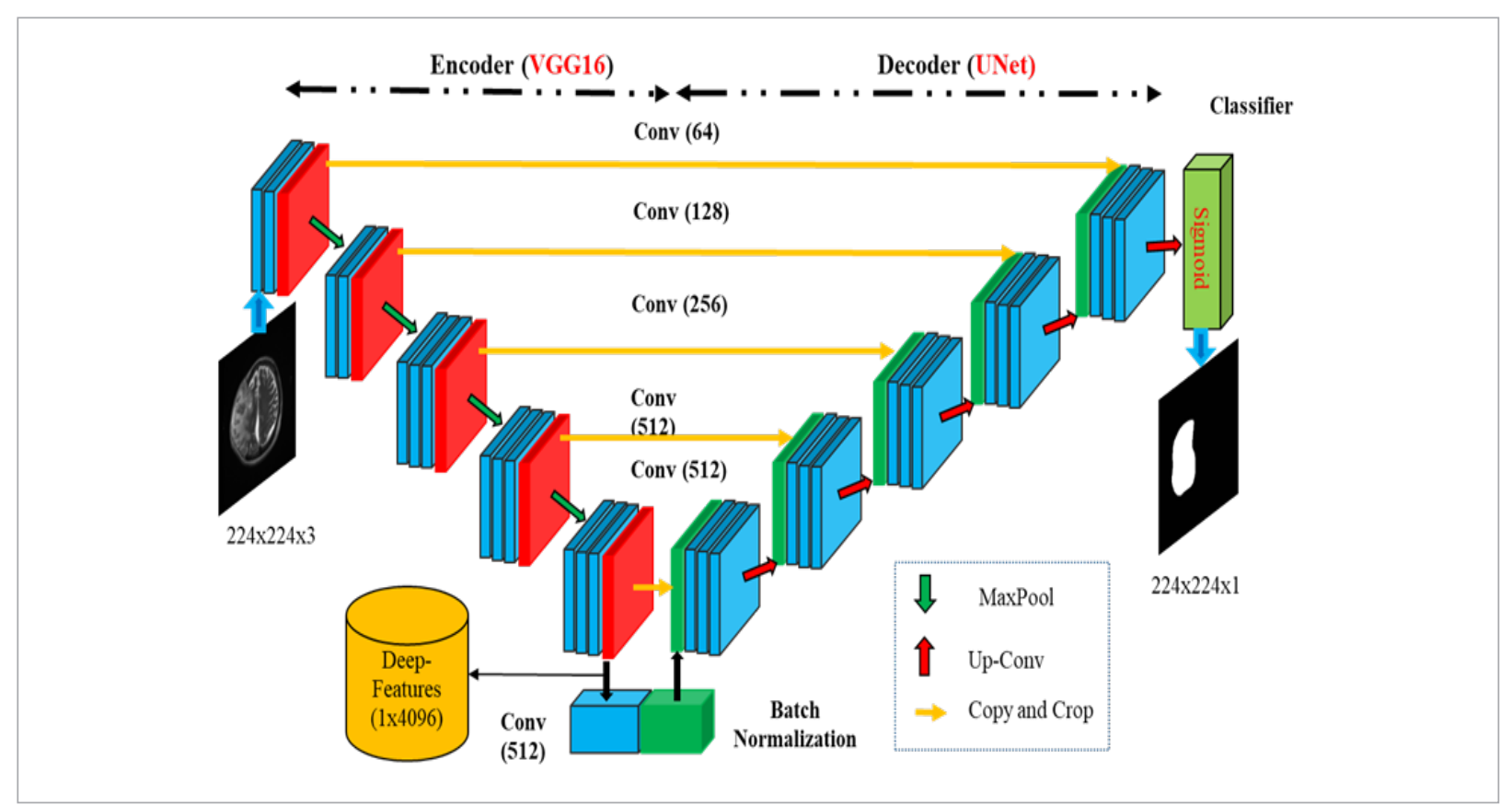

traditional VGG16 scheme with operations, such as convolution, ReLU and max pool.

The outcome of the encoder section presents the DF with a value of $1 \times 4096$ which is then stored separately for further assessment. The extracted DF are then normalized and processed with the UNet decoder unit. The number of layers in encoder as well as decoder is unique (5 layers) and the final layer of decoder is then given to a SoftMax classifier which implements a binary classification to separate the BT with background using a Signoid-activation-function. This BT segment is then considered to extract the GLCM features.

\subsection{Deep-Features}

The total number of DF extracted is very large (1x4096) and hence three fully-connected-layers (FCL) with $50 \%$ dropout are considered to get a reduced feature with dimension $1 \times 1024$. All these features are then sorted based on their rank and later a FA assisted feature selection is then implemented to overcome the over-fitting problem, commonly originate in binary classification. Equation (1) presents the feature-vector attained from VGG-UNet and
Equation (2) depicts the feature-vector after the FCL dropout.

$$
\begin{aligned}
& F V_{V G G-U n e t(1 \times 4096)}=V G G-\text { Unet }_{(1,1)}, \\
& V G G-\text { Unet }_{(1,2)}, \ldots, \\
& V G G-\text { Unet }_{(1,4096)}
\end{aligned}
$$

$\mathrm{FV}_{\mathrm{DF}}(1 \times 1024)=\mathrm{DF}_{(1,1)}, \mathrm{DF}_{(1,2)}, \ldots, \mathrm{DF}_{(1,1024)}$.

\subsection{Handcrafted-Features}

The earlier works $[11,16,17,23]$ confirmed that the combination of DF and HF (DF+HF) will improve the performance of deep-learning system. In this work, the essential HF from the brain MRI slices are mined using the well known methods such as GLCM $[3,10$, 29], $\mathrm{Hu}[3,10,30]$ and LBP [31, 32]. The GLCM is widely adopted due to its superior performance and the essential GLCM parameters of the MRI slices are extracted from the segmented BT by VGG-UNet. Similar procedure is implemented to extract the $\mathrm{Hu}$ moments. Equation (3) and Equation (4) present the extracted GLCM and Hu features. 


$$
\begin{aligned}
& H F 1_{G \operatorname{LM}(1 \times 25)}=\operatorname{GLCM} M_{(1,1)}, G L C M_{(1,2)}, \ldots, \\
& \operatorname{GLCM}_{(1,25)}
\end{aligned}
$$

$$
\mathrm{HF}^{2} \mathrm{Hu}(1 \times 7)=\mathrm{Hu}_{(1,1)}, \mathrm{Hu}_{(1,2)}, \ldots, \mathrm{Hu}_{(1,7)} \text {. }
$$

The LBP provides the important information regarding the gray-scale picture under assessment and the LBP with different weight discussed in [32] is adopted to extract the pixel information of MRI slices with GBM/LGG. In this work, the weights, such as $\mathrm{W}=1,2$, 3 , and 4 are considered to enhance the image and from each image, 1x59 number of features are extracted. The LBP features considered in this work are depicted in Equation (5):

$$
\begin{aligned}
& H F 3_{L B P(1 \times 236)}=L B P 1_{(1,59)}+L B P 2_{(1,59)} \\
& +L B P 3_{(1,59)}+L B P 4_{(1,59)} .
\end{aligned}
$$

The total number of HF collected with GLCM, Hu and LBP is shown in Equation (6);

$$
\begin{aligned}
& H F_{(1 \times 268)}=H F 1_{G \operatorname{LCM}(1 \times 25)}+H F 2_{H \mathrm{u}(1 \times 7)} \\
& +H F 3_{L B P(1 \times 236)} .
\end{aligned}
$$

\subsection{Firefly-Algorithm Based Feature Selection and Serial Fusion}

The feature reduction process plays a vital role during in ML and DL based classification and to avoid the over-fitting problem, it is necessary to identify the dominant features using an appropriate approach. The feature reduction can be implemented using traditional statistical approaches (Student's t-test) $[3,12]$ and heuristic algorithm assisted techniques [17, 22]. In this work, the feature reduction for DF and HF are implemented using the FA algorithm and the reduced features are then serially combined as discussed in [33].

The FA feature selection is implemented as follows;

Let us consider there exist feature vectors $\mathrm{FV}_{\mathrm{GBM}}$ and; let this vector is with a value of $\mathrm{FV}_{\mathrm{GBM}}, \mathrm{FV}_{\mathrm{LGG}} \in\{0,1\}^{\mathrm{N}}$. The FA then performs a feature wise assessment and computes the Hamming-Distance (HD) as in Equation (7):

$$
\mathrm{HD}\left(\mathrm{FV}_{\mathrm{GBM}}, \mathrm{FV}_{\mathrm{LGG}}\right)=\sum_{\mathrm{a}=1}^{\mathrm{N}}\left|\mathrm{FV}_{\mathrm{GBMa}}-\mathrm{FV}_{\mathrm{LGGa}}\right|,
$$

where $\mathrm{N}=1024$ in $\mathrm{DF}$ and $\mathrm{N}=268$ in $\mathrm{HF}$.
The difference in val1ues between features is expressed as in Equation (8);

$$
\operatorname{Diff}\left(F V_{G B M}, F V_{L G G}\right)=\sum_{a=1}^{N}\left(F V_{G B M a}-F V_{L G G a}\right) .
$$

The fitness function then assigned as in Equation (9):

Fitnessvalue $=H D_{\max }\left(F V_{G B M}, F V_{L G G}\right)=$

$\sum_{a=1}^{N}\left|F V_{G B M a}-F V_{L G G a}\right|$

The $\mathrm{HD}_{\max }\left(\mathrm{FV}_{\mathrm{GBM}}, \mathrm{FV}_{\mathrm{LGG}}\right)$ is then evaluated to decide the features and if $\mathrm{HD}_{\max }\left(\mathrm{FV}_{\mathrm{GBM}}, \mathrm{FV}_{\mathrm{LGG}}\right) \approx 0$, then the particular feature is discarded and if $H D_{\max }\left(F V_{G B M}, F V_{L G G}\right) \neq 0$, then the corresponding feature is selected and a new feature vector is formed. The proposed procedure is graphically presented in Figure 5 and the FA with optimally assigned parameters will help to identify the new feature vector.

\section{Figure 5}

Firefly algorithm based dominant feature selection

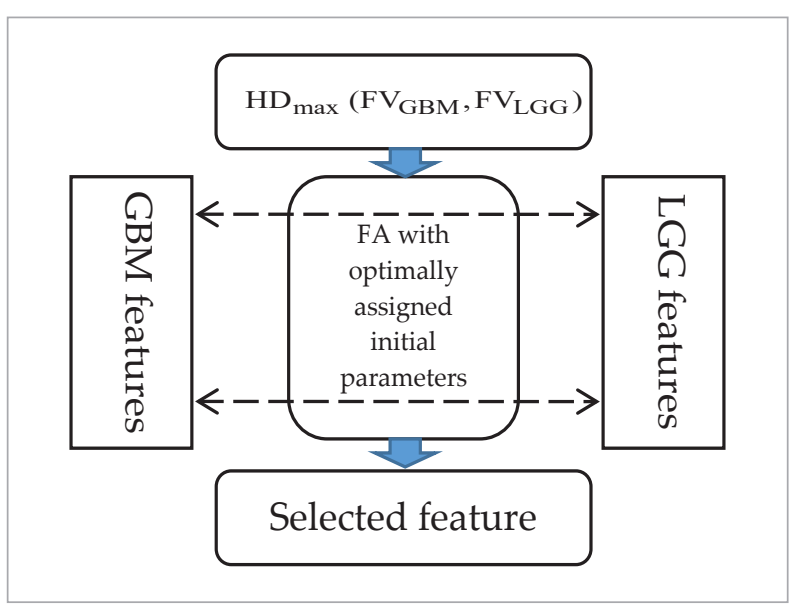

In this work, the FA with Brownian-Distribution is considered and other essential parameters are assigned as follows; number of fireflies $=30$, search dimension $=$ total features, iterations $=2500$ and other FA parameter information can be accessed from [34].

The FA based feature selection helped to get DF vector with a dimension of $1 \times 427$ and HF with a dimension of $1 \times 193$. These features are serially combined to get a new fused-feature-vector (FFV) depicted in Equation (10). This FFV is then considered to train, 
test and validate the binary classifiers considered in the developed CADD unit.

$$
\mathrm{FFV}_{(1 \times 620)}=\mathrm{DF}_{(1 \times 427)}+\mathrm{HF}_{(1 \times 193)}
$$

\subsection{Classifier Implementation and Performance Validation}

The performance of the medical data assessment using the developed CADD depends on the employed classifiers. Binary classification is implemented in the proposed work to classify the MRI slices into GBM/LGG class for benchmark as well as clinical data. To achieve this task, the classifiers existing in the literature, such as SoftMax, SVM with various kernels (Linear, RBF, and Cubic) [3, 12, 33, 35-37], DA (Linear, and Quadratic) $[12,33]$ and KNN (Fine, and Cubic) $[12,33]$ are employed to accomplish the task. The earlier research also presents the similar medical image assessment tasks which implemented classifiers [38-41].

The performance of the classifier is then assessed by recording the confusion-matrix values, such as true-positive (TP), true-negative (TN), false-positive (FP), false-negative (FN), accuracy (ACC), precision (PRE), sensitivity (SEN), specificity (SPE) and negative predictive value (NPV) $[3,11]$. Based on these values, the performance of proposed CADD with a chosen binary classifier is confirmed.

\section{Experimental Results}

This section presents the experimental outcome attained with the proposed work. The experimental investigation is implemented using a workstation with
Intel I5 2.5GHz processor with 16GB RAM and 2GB VRAM equipped with MATLAB ${ }^{\circ}$.

Initially, the essential number of MRI slices is extracted from benchmark as well as the clinical dataset as discussed in Table 1 and every image is then resized into $224 \times 224 \times 3$ pixels to implement the selected CNN scheme. Initially, the VGG-UNet is implemented to extract the BT segment from the considered test images. The VGG-UNet depicted in Figure 4 is initially trained for the considered image data using the original and augmented benchmark images and after the training, the segmentation performance of VGG-UNet is then validated using the benchmark and clinical grade MRI slices. Figure 6 depicts the result attained for GBM class clinical MRI and in this image Figure 6(a) to (d) shows the various layer outputs of VGG-UNet and Figure 6(e) and (f) depicts the identified section (heatmap) and extracted section by the decoder's SoftMax unit. Similar result is then attained with other validation images considered in this work.

The encoder section of the VGG-UNet also helps to extract the essential DF with a dimension of $1 \mathrm{x} 4096$ and this feature value is then initially reduced to $1 \times 1024$ features using the FCL and further this DF is reduced to $1 \times 427$ using the FA based feature reduction technique. After collecting the essential DF vector, the necessary HF are then collected with GLCM and Hu moments. To get the LBP from the test images, various weight values ( $W=1,2,3$ and 4 ) are implemented and the corresponding outcome attained with GBM and LGG class images are presented in Figure 7. From every LBP image, a feature value with dimension 1x59 is then extracted and all these features are combined together to get the essential feature value for LBP (1x236).

\section{Figure 6}

Vaious level output attained with VGG-UNet for GBM class test image. (a)-(d) Image outcomes attained in various layers of VGG-UNet, (e) Heatmap for the identified tumour section, (f) Segmented tumour using VGG-UNet

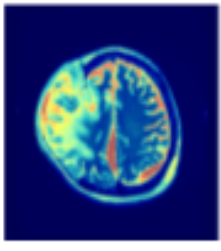

(a)

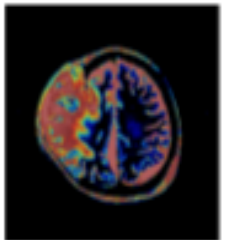

(b)

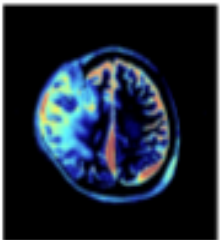

(c)

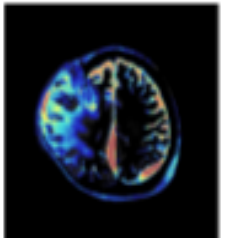

(d)

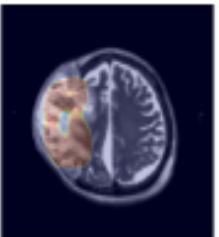

(e)

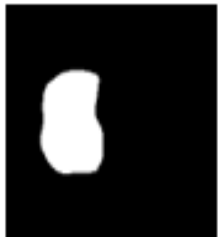

(f) 


\section{Figure 7}

LBP treated brain MRI slices with chosen weights

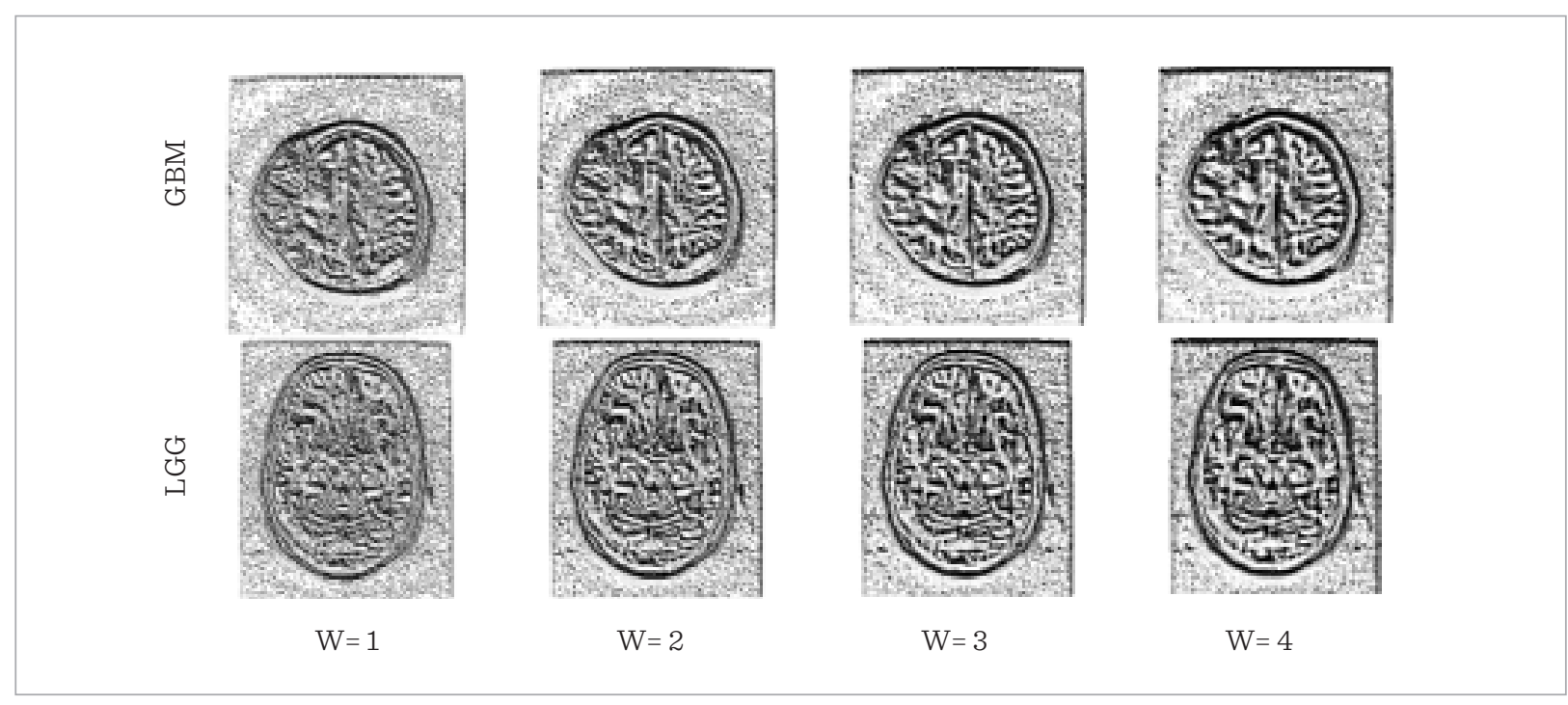

Finally, the $\mathrm{DF}+\mathrm{HCF}$ is implemented and the FFV is then considered to authenticate the classifiers and based on the attained confusion-matrix parameters, the eminence of the proposed CADD is confirmed. During this classification task, a 10-fold cross validation is employed and the best result which is attained during the 10 trial is adopted as the best result by the considered classifier.

\section{Discussion}

This section presents the merit of the proposed scheme on the considered image database. The performance of CADD is separately validated on the considered image datasets, initially CADD's performance is tested using the TCIA database and the attained result is presented in Table 2 for $\mathrm{DF}$ alone and $\mathrm{DF}+\mathrm{HF}$.

The result presented in Table 2 depicts the results achieved with the binary classifier and the presented result confirms that the SVM classifier outperforms other classifier units, such as SoftMax, DA and KNN considered in this study. Figure 8 presents the Glyphplot to demonstrate the classifier performance with $\mathrm{DF}$ along and $\mathrm{DF}+\mathrm{HF}$ and from this figure, it can be noted that, the overall performance offered by the SVM-RBF in the case of DF (Figure 8(a)) and the performance by SVM-Cubic is better for DF+HF. This outcome confirms that, the classification accuracy of the $\mathrm{CADD}$ is improved due to the $\mathrm{DF}+\mathrm{HF}$ compared to the classification accuracy by $\mathrm{DF}$ alone.

Related practice is then repeated with the clinical MRI dataset and the attained results are presented in Table 3. From Table 3, it can be noted that the SVM-Cubic classifier helped to achieve better classification accuracy with $\mathrm{DF}$ and $\mathrm{DF}+\mathrm{HF}$ feature vectors. The confusion matrix presented in Figure 9 (a) and (b) also confirms the eminence of the SVM-Cubic classifier compared to other methods. Further, the Receiver Operating Characteristic (ROC) curve presented in Figure 9(c) confirms that the SVM-Cubic offers better result with $\mathrm{DF}+\mathrm{HF}$ compared to other SVM, DA, KNN and SoftMax classifiers considered in this work.

The overall performance of the CADD is then presented in Figure 10 using the Glyph-plot and this figure also confirms that the performance of the SSM-Cubic is better with the clinical images for $\mathrm{DF}$ and $\mathrm{DF}+\mathrm{HF}$. These results confirm that, proposed CNN based segmentation and SVM classification is clinically noteworthy and the developed CADD can be considered to 
Table 2

The disease detection performance of CADD with TCIA database

\begin{tabular}{|c|c|c|c|c|c|c|c|c|c|c|}
\hline Features & Classifier & $\mathrm{TP}$ & $\mathrm{FN}$ & $\mathrm{TN}$ & FP & $\mathrm{ACC}$ & PRE & SEN & SPE & NPV \\
\hline \multirow{8}{*}{ 卧 } & SoftMax & 219 & 21 & 223 & 17 & 92.08 & 92.80 & 91.25 & 92.92 & 91.39 \\
\hline & SVM-Linear & 217 & 23 & 216 & 24 & 90.21 & 90.04 & 90.42 & 90.00 & 90.38 \\
\hline & SVM-RBF & 226 & 14 & 222 & 18 & 93.33 & 92.62 & 94.17 & 92.50 & 94.07 \\
\hline & SVM-Cubic & 221 & 19 & 225 & 15 & 92.92 & 93.64 & 92.08 & 93.75 & 92.21 \\
\hline & DA-Linear & 218 & 22 & 221 & 19 & 91.46 & 91.98 & 90.83 & 92.08 & 90.95 \\
\hline & DA-Quadratic & 220 & 20 & 218 & 22 & 91.25 & 90.91 & 91.67 & 90.83 & 91.60 \\
\hline & KNN-Fine & 224 & 16 & 222 & 18 & 92.92 & 92.56 & 93.33 & 92.50 & 93.28 \\
\hline & KNN-Cubic & 217 & 23 & 219 & 21 & 90.83 & 91.18 & 90.42 & 91.25 & 90.50 \\
\hline \multirow{8}{*}{ 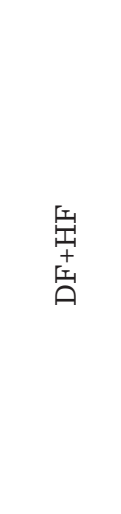 } & SoftMax & 230 & 10 & 227 & 13 & 95.21 & 94.65 & 95.83 & 94.58 & 95.78 \\
\hline & SVM-Linear & 228 & 12 & 223 & 17 & 93.96 & 93.06 & 95.00 & 92.92 & 94.89 \\
\hline & SVM-RBF & 225 & 15 & 231 & 9 & 95.00 & 96.15 & 93.75 & 96.25 & 93.90 \\
\hline & SVM-Cubic & 230 & 10 & 231 & 9 & 96.04 & 96.23 & 95.83 & 96.25 & 95.85 \\
\hline & DA-Linear & 231 & 9 & 223 & 17 & 94.58 & 93.15 & 96.25 & 92.92 & 96.12 \\
\hline & DA-Quadratic & 224 & 16 & 230 & 10 & 94.58 & 95.73 & 93.33 & 95.83 & 93.50 \\
\hline & KNN-Fine & 233 & 7 & 226 & 14 & 95.63 & 94.33 & 97.08 & 94.17 & 97.00 \\
\hline & KNN-Cubic & 228 & 12 & 229 & 11 & 95.21 & 95.40 & 95.00 & 95.42 & 95.02 \\
\hline
\end{tabular}

Figure 8

Glyph-plot generated using the classifier performance for TCIA image database

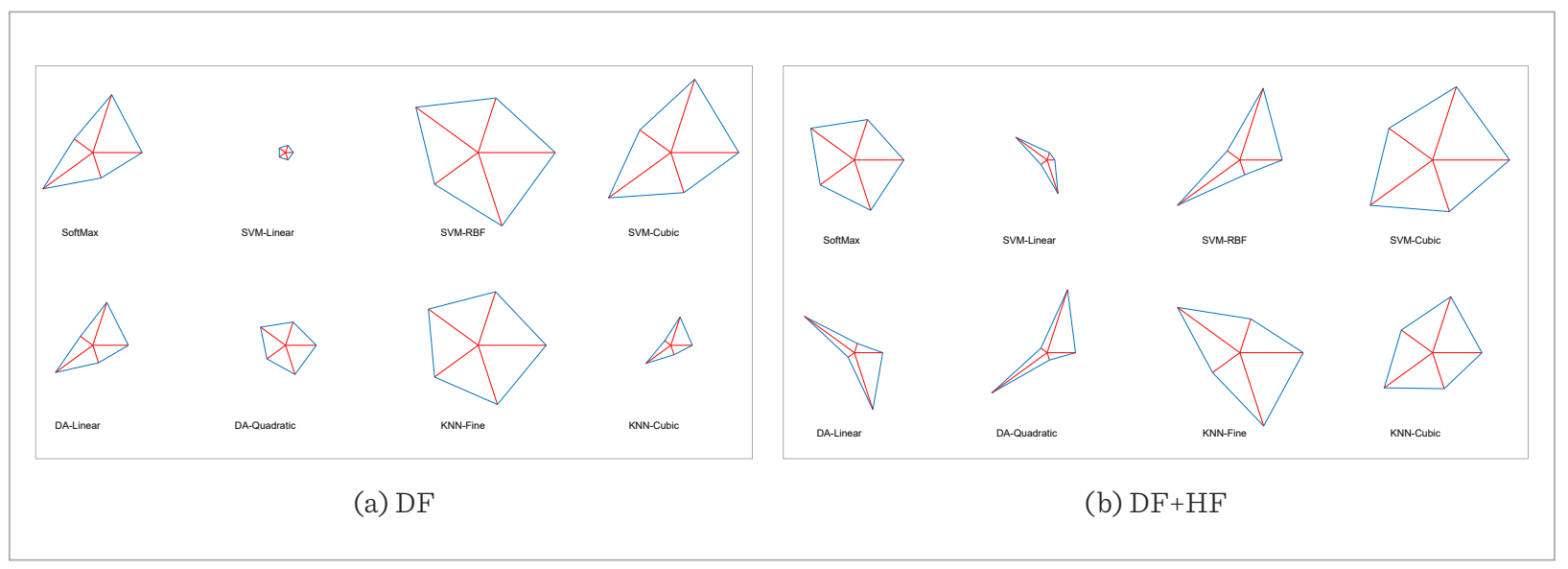


Table 3

Brain tumour detection performance of CADD with clinical images

\begin{tabular}{|c|c|c|c|c|c|c|c|c|c|c|}
\hline Features & Classifier & $\mathrm{TP}$ & FN & $\mathrm{TN}$ & FP & $\mathrm{ACC}$ & PRE & SEN & SPE & NPV \\
\hline \multirow{7}{*}{ 卧 } & SoftMax & 82 & 8 & 84 & 6 & 92.22 & 93.18 & 91.11 & 93.33 & 91.30 \\
\hline & SVM-Linear & 85 & 5 & 81 & 9 & 92.22 & 90.43 & 94.44 & 90.00 & 94.19 \\
\hline & SVM-RBF & 83 & 7 & 80 & 10 & 90.56 & 89.25 & 92.22 & 88.89 & 91.95 \\
\hline & SVM-Cubic & 85 & 5 & 83 & 7 & 93.33 & 92.39 & 94.44 & 92.22 & 94.32 \\
\hline & DA-Linear & 81 & 9 & 86 & 4 & 92.78 & 95.29 & 90.00 & 95.56 & 90.53 \\
\hline & DA-Quadratic & 83 & 7 & 85 & 5 & 93.33 & 94.32 & 92.22 & 94.44 & 92.39 \\
\hline & KNN-Fine & 84 & 6 & 82 & 8 & 92.22 & 91.30 & 93.33 & 91.11 & 93.18 \\
\hline \multirow{9}{*}{ 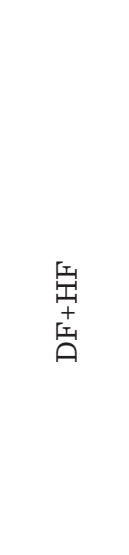 } & KNN-Cubic & 86 & 4 & 81 & 9 & 92.78 & 90.53 & 95.56 & 90.00 & 95.29 \\
\hline & SoftMax & 85 & 5 & 85 & 5 & 94.44 & 94.44 & 94.44 & 94.44 & 94.44 \\
\hline & SVM-Linear & 86 & 4 & 88 & 2 & 96.67 & 97.73 & 95.56 & 97.78 & 95.65 \\
\hline & SVM-RBF & 88 & 2 & 87 & 3 & 97.22 & 96.70 & 97.78 & 96.67 & 97.75 \\
\hline & SVM-Cubic & 89 & 1 & 89 & 1 & 98.89 & 98.88 & 98.88 & 98.88 & 98.88 \\
\hline & DA-Linear & 86 & 4 & 85 & 5 & 95.00 & 94.51 & 95.56 & 94.44 & 95.51 \\
\hline & DA-Quadratic & 89 & 1 & 88 & 2 & 98.33 & 97.80 & 98.89 & 97.78 & 98.88 \\
\hline & KNN-Fine & 88 & 2 & 89 & 1 & 98.33 & 98.88 & 97.78 & 98.89 & 97.80 \\
\hline & KNN-Cubic & 88 & 2 & 88 & 2 & 97.78 & 97.78 & 97.78 & 97.78 & 97.78 \\
\hline
\end{tabular}

Figure 9

SVM-Cubic performance for clinical MRI database

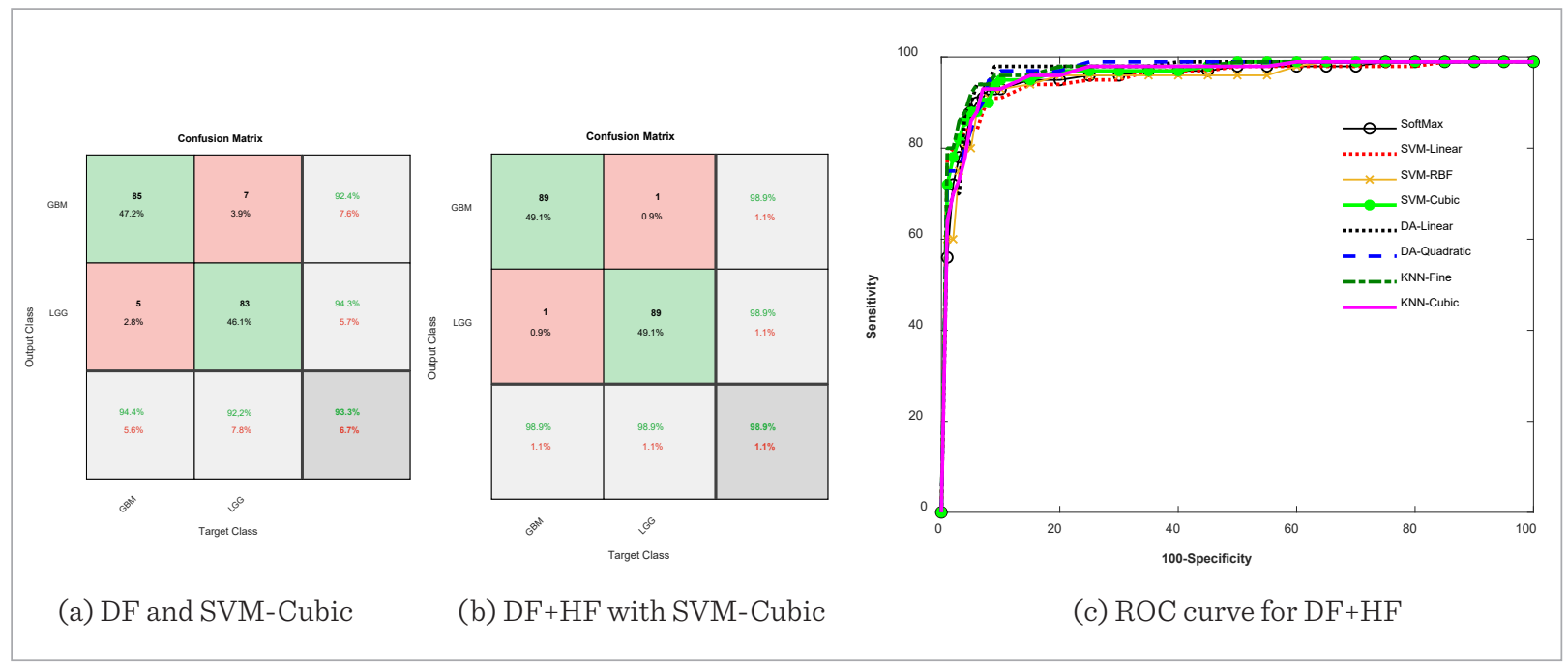




\section{Figure 10}

Glyph-plot generated using the classifier performance for clinical MRI database

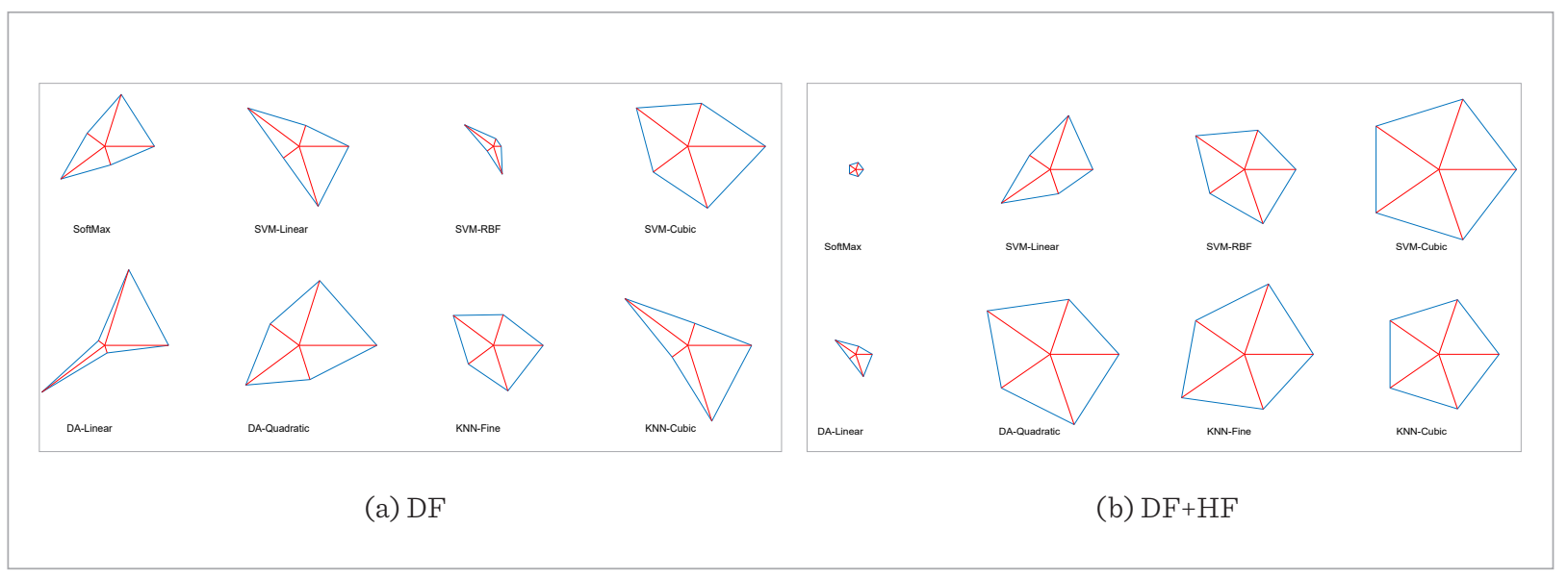

examine the clinical level MRI slices collected from real patients.

The main contribution of the proposed work is that, it has developed a CNN assisted CADD system which is used to detect and classify the BT into GBM/LGG with better accuracy. The proposed approach helped to attain a classification accuracy of $>98 \%$ on the clinically collected images. The future extent of this work includes; (i) Improving the performance of VGG-UNet using VGG19 scheme, (ii) Comparing the performance of VGG-UNet with other CNN segmentation methods existing in the literature and (iii) Testing and validating the performance of proposed CADD using the brain MRI of modalities, such as $\mathrm{T} 1, \mathrm{~T} 1 \mathrm{C}$ and Flair.

\section{Conclusion}

The chief intention of this research is to develop a CNN supported CADD unit to categorize the brain MRI slices into GBM/LGG class. This work developed the CADD system using; (i) VGG-UNet assisted segmentation and DF extraction, (ii) HF extraction using GLCM, Hu and LBP, (iii) Dominant feature selection using FA, and (iv) Feature fusion and validation using various binary classifiers. The performance of the proposed CADD is separately tested using the TCIA and clinical MRI dataset and the result attained with the proposed scheme substantiate that, proposed work assist to accomplish better classification accuracy on the TCIA as well as the benchmark dataset. The classifier attained with the clinical grade MRI using the $\mathrm{DF}$ and $\mathrm{DF}+\mathrm{HF}$ with a ten-fold cross validation confirms that, proposed CADD system offers better outcome with SVM-Cubic classifier compared to other binary classifiers. In future, this CADD can be adopted to inspect the clinically collected brain MRI slices of T2 modality.

\section{Acknowlegment}

This research was supported by the MSIT(Ministry of Science and ICT), Korea, under the ICAN(ICT Challenge and Advanced Network of HRD) program(IITP-2021-2020-0-01832) supervised by the IITP(Institute of Information \& Communications Technology Planning \& Evaluation) and the Soonchunhyang University Research Fund.

\section{Special Thanks}

The authors of this article would like to acknowledge the help and support by Dr. K. Arvind Karthik, Managing Director and Radiologist, M/S. Proscans Diagnostics Pvt. Ltd., Chennai, Tamil Nadu, India for providing the clinical brain MRI for experimental investigation. 


\section{References}

1. Acharya, U. R., Fernandes, S. L., Weikoh, J. E., Ciaccio, E. J., Fabell, M. K. M., Tanik, U. J., Rajinikanth, V., Yeong, C. $\mathrm{H}$. Automated Detection of Alzheimer's Disease Using Brain MRI Images-A Study with Various Feature Extraction Techniques. Journal of Medical Systems, 2019, 43(9), 302. https://doi.org/10.1007//s10916-019-1428-9

2. Bakiya, A., Kamalanand, K., Rajinikanth, V., Nayak, R., Kadry, S. Deep Neural Network Assisted Diagnosis of Time-Frequency Transformed Electromyograms. Multimedia Tools and Applications, 2020, 79(15), 11051-67. https://doi.org/10.1007//s11042-018-6561-9

3. Balakrishna, C., Dadashzadeh, S., Soltaninejad, S. Automatic Detection of Lumen and Media in the Ivus Images Using U-net with Vgg16 Encoder. Arxiv Preprint Arxiv:1806.07554.2018.

4. Bhandary, A., Prabhu, G., Rajinikanth, V., Thanaraj, K., Satapathy, S., Robbins, D. E., Shasky, C., Zhang, Y.-D., Tavares, J., M, R. S., Raja, N. S. M. Deep-Learning Framework to Detect Lung Abnormality-A Study With Chest $\mathrm{X}$-ray and Lung CT Scan Images. Pattern Recognition Letters, 2020, 129, 271-8.https://doi.org/10.1016/j.patrec.2019.11.013

5. Clark, K., Vendt, B., Smith, K., Freymann, J., Kirby, J., Koppel, P., Moore, S., Phillips, S., Maffitt, D., Pringle, M., Tarbox, L., Prior, F. The Cancer Imaging Archive (TCIA): Maintaining and Operating a Public Information Repository. Journal of Digital Imaging, 2013, 26(6), 1045-57. https://doi.org/10.1007/s10278-013-9622-7

6. Dey, N., Rajinikanth, V., Shi, F., Tavares, J., Moraru, L., Karthik, K. A., Kamalanand, K., Emmanuel, C. Social-Group-Optimization Based Tumor Evaluation Tool for Clinical Brain MRI of Flair/Diffusion-Weighted Modality. Biocybernetics and Biomedical Engineering, 2019, 39(3), 843-56. https://doi.org/10.1016/j.bbe.2019.07.005

7. Fernandes, S., Tanik, U., Rajinikanth, V., Karthik, K. A Reliable Framework for Accurate Brain Image Examination and Treatment Planning Based on Early Diagnosis Support for Clinicians. Neural Computing and Applications, 2020, 32(20), 15897-908. https://doi. org/10.1007/s00521-019-04369-5

8. Godse, R., Bhat, S. Combined Morphology and SVMBased Fault Feature Extraction Technique for Detection and Classification of Transmission Line Faults. Turkish Journal of Electrical Engineering \& Computer Sciences, 2020, 28(5), 2768-88. https://doi.org/10.3906/elk-1912-7

9. Gudigar, A., Raghavendra, U., Devasia, T., Nayak, K., Danish, S., Kamath, G., Samanth, J., Pai, U. M., Naya, V., Tan,
R. S., Ciaccio, E. J., Acharya, U. R. Global Weighted LBP Based Entropy Features for the Assessment of Pulmonary Hypertension. Pattern Recognition Letters, 2019, 125, 35-41. https://doi.org/10.1016/j.patrec.2019.03.027

10. Gülgün, O., Hamza, E. Classification Performance Comparisons of Deep Learning Models in Pneumonia Diagnosis Using Chest X-ray Images. Turkish Journal of Engineering, 2020, 4(3), 129-141.https://doi.org/10.31127/ tuje.652358

11. Hussain, U., Khan, M., Lali, I., Javed, K., Ashraf, I., Tariq, J., Ali, H., Din, A. A Unified Design of Aco and Skewness Based Brain Tumor Segmentation and Classification from MRI Scans. Journal of Control Engineering and Applied Informatics, 2020, 22(2), 43-55.

12. Jafarpour, S., Sedghi, Z., Amirani, M. A Robust Brain MRI Classification with GLCM Features. International Journal of Computer Applications, 2012, 37(12), 1-5. https://doi.org/10.5120/4735-6872.

13. Jiao, L., Huo, L., Hu, C., Tang, P. Refined Unet V2: Endto-End Patch-Wise Network for Noise-Free Cloud and Shadow Segmentation. Remote Sensing, 2020, 12(21), 3530. https://doi.org/10.3390/rs12213530

14. Kallenberg, K., Bock, H., Helms, G., Jung, K., Wrede, A., Buhk, J.-H., Giese, A., Frahm, J., Strik, H., Dechent, P., Knauth, M. Untreated Glioblastoma Multiforme: Increased Myo-inositol and Glutamine Levels in the Contralateral Cerebral Hemisphere at Proton MRI Spectroscopy. Radiology, 2009, 253(3), 805-812. https://doi. org/10.1148/radiol.25330'71654

15. Karimi, D., Dou, H., Warfield, S. K., Gholipour, A. Deep Learning with Noisy Labels: Exploring Techniques and Remedies in Medical Image Analysis. Medical Image Analysis, 2020, 65, 101759. https://doi.org/10.1016/j. media.2020.101759

16. Ke, Q., Zhang, J., Wei, W., Damaševičius, R., Woźniak, M. Adaptive Independent Subspace Analysis of Brain MagneticResonance Imaging Data. Ieee Access, 2019, 7,1225212261. https://doi.org/10.1109/ACCESS.2019.2893496

17. Khan, M., Ashraf, I., Alhaisoni, M., Damaševičius, R., Scherer, R. Et Al. Multimodal Brain Tumor Classification Using Deep Learning and Robust Feature Selection: A Machine Learning Application for Radiologists. Diagnostics, 2020, 10(8), 565. https://doi.org/10.3390/ diagnostics10080565

18. Khan, M., Kadry, S., Alhaisoni, M., Nam, Y., Zhang, Y., Rajinikanth, V., Sarfraz, M. S. Computer-Aided Gastrointestinal Diseases Analysis from Wireless Capsule 
Endoscopy: A Framework of Best Features Selection. IEEE Access, 2020, 8, 132850-132859. https://doi. org/10.1109/ACCESS.2020.3010448

19. Khan, M., Rubab, S., Kashif, A., Sharif, M., Muhammad, N., Shah, J. H., Zhang, Y.-D., Satapathy, S. C. Lungs Cancer Classification from CT Images: An Integrated Design of Contrast Based Classical Features Fusion and Selection. Pattern Recognition Letters, 2020, 129, 7785. https://doi.org/10.1016/j.patrec.2019.11.014

20. Khan, M. A., Sarfraz, M. S., Alhaisoni, M., Albesher, A. A., Wang, S., Ashraf, I.. Stomachnet: Optimal Deep Learning Features Fusion for Stomach Abnormalities Classification. IEEE Access, 2020. https://doi.org/10.1109/ ACCESS.2020.3034217

21. Khan, S., Nazir, M., Khan, M., Saba, T., Javed, K., Rehman, A., Akram, T., Awais, M. Lungs Nodule Detection Framework from Computed Tomography Images Using Support Vector Machine. Microscopy Research and Technique, 2019, 82(8), 1256-1266.https://doi.org/10.1002/ jemt.23275

22. Louis, D., Perry, A., Reifenberger, G., Deimling, V., Figarella-Branger, D., Cavenee, W. K., Ohgaki, H., Wiestler, O. D., Kleihues, P., Ellison, D. W. The 2016 World Health Organization Classification of Tumors of the Central Nervous System: A Summary. Acta Neuropathologica, 2016, 131(6), 803-820. https://doi.org/10.1007/s00401-016-1545-1

23. Nazar, U., Khan, M., Lali, I., Lin, H., Ali, H., Ashraf, I., T., J. Review of Automated Computerized Methods for Brain Tumor Segmentation and Classification. Current Medical Imaging, 2020, 16(7), 823-34. https://doi.org/1 $0.2174 / 1573405615666191120110855$

24. Öztürk, Ş. Stacked Auto-Encoder Based Tagging with Deep Features for Content-Based Medical Image Retrieval. Expert Systems with Applications, 2020, 161, 113693. https://doi.org/10.1016/j.eswa.2020.113693

25. Pedano, N., Flanders, A., Scarpace, L., Mikkelsen, T., Eschbacher, J. M., Hermes, B., Ostrom, Q. Radiology Data from the Cancer Genome Atlas Low Grade Glioma [tcga-lgg] Collection. The Cancer Imaging Archive, 2016. http://doi.org/10.7937/k9/tcia.2016.14ltd3tk.

26. Połap, D. An Adaptive Genetic Algorithm as a Supporting Mechanism for Microscopy Image Analysis in a Cascade of Convolution Neural Networks. Applied Soft Computing, 2020, 97, 106824. https://doi.org/10.1016/j. asoc.2020.106824

27. Połap, D. Analysis of Skin Marks Through the use of Intelligent Things. IEEE Access, 2019; 7, 149355-149363. https://doi.org/10.1109/ACCESS.2019.2947354
28. Pugalenthi, R., Rajakumar, M., Ramya, J., Rajinikanth, V. Evaluation and Classification of the Brain Tumor MRI Using Machine Learning Technique. Journal of Control Engineering and Applied Informatics, 2019, 21(4), 12-21.

29. Qureshi, S., Karilla, S., Vanichayobon, S. Gacnn Sleeptunenet: A Genetic Algorithm Designing the Convolutional Neural Network Architecture for Optimal Classification of Sleep Stages from a Single EEG Channel. Turkish Journal of Electrical Engineering \& Computer Sciences, 2019, 27(6), 4203-419. https://doi. org/10.3906/elk-1903-186

30. Raja, S., Rajinikanth, V., Latha, K. Otsu Based Optimal Multilevel Image Thresholding Using Firefly Algorithm. Modelling and Simulation in Engineering, 2014. https://doi.org/10.1155/2014/794574

31. Rajinikanth, V., Raj, J., Thanaraj, K., Naik, G. A Customized Vgg19 Network with Concatenation of Deep and Handcrafted Features for Brain Tumor Detection. Applied Sciences, 2020, 10(10), 3429. https://doi. org/10.3390/app10103429

32. Rajinikanth, V., Satapathy, S. Segmentation of Ischemic Stroke Lesion in Brain MRI Based on Social Group Optimization and Fuzzy-tsallis Entropy. Arabian Journal for Science and Engineering, 2018, 43(8), 4365-478. https://doi.org/10.1007/s13369-017-3053-6

33. Rehman, A., Khan, M., Saba, T., Mehmood, Z., Tariq, U., Ayesha, N. Microscopic Brain Tumor Detection and Classification Using 3D CNN and Feature Selection Architecture. Microscopy Research and Technique, 2020. https://doi.org/10.1002/jemt.23597

34. Ronneberger, O., Fischer, P., Brox, T. U-net: Convolutional Networks for Biomedical Image Segmentation. In: Navab, N., Hornegger, J., Wells, W., Frangi, A. (Eds.) Medical Image Computing and Computer-Assisted Intervention - Miccai 2015. Miccai 2015. Lecture Notes in Computer Science, 2015, 9351. https://doi. org/10.1007/978-3-319-24574-4_28

35. Saba, T., Mohamed, A., El-affendi, M., Amin, J., Sharif, M. Brain Tumor Detection Using Fusion of Hand Crafted and Deep Learning Features. Cognitive Systems Research, 2020, 59, 221-230. https://doi.org/10.1016/j. cogsys.2019.09.007

36. Sabhara, R., Lee, C., Lim, K. Comparative Study of Hu Moments and Zernike Moments in Object Recognition. SMARTCR, 2013, 3(3), 166-173. https://doi.org/10.6029/ smartcr.2013.03.003

37. Scarpace, L., Mikkelsen, T., Cha, S., Rao, S., Tekchandani, S., et al. Radiology Data from the Cancer Genome 
Atlas Glioblastoma Multiforme [tcga-gbm] Collection. The Cancer Imaging Archive, 2016. http://doi. org/10.7937/k9/tcia.2016.rnyfuye9

38. Sharif, M., L, J., Khan, M., Salee, M. Active Deep Neural Network Features Selection for Segmentation and Recognition of Brain Tumors Using MRI Images. Pattern Recognition Letters, 2020, 129, 181-189. https://doi. org/10.1016/j.patrec.2019.11.019

39. Vlis, V., Hoeben, A., Beckervordersandforth, J., Ackermans, L., Eekers, D., Wennekes, R. M. J., Schijns, O. E. M. G. Impact of the Revised Who Classification of Dif- fuse Low-Grade Glioma on Clinical Decision Making: A Case Report. Surgical Neurology International, 2017, 8. https://doi.org/10.4103/sni.sni_166_17'

40. Yasrab, R. ECRU: An Encoder-Decoder Based Convolution Neural Network (CNN) for Road-Scene Understanding. Journal of Imaging, 2018, 10, 116. https://doi. org/10.3390/jimaging4100116

41. Yushkevich, P., Piven, J., Hazlett, H., Smith, R., Ho, S. User-Guided 3D Active Contour Segmentation of Anatomical Structures: Significantly Improved Efficiency and Reliability. Neuroimage, 2006, 31(3), 1116-128. https://doi.org/10.1016/j.neuroimage.2006.01.015 